\title{
Clinical Relevance of IgE to Profilin and/or Polcalcin in Pollen-Sensitized Patients
}

\author{
Marion San Nicoló Thomas Braun Katharina Eder Alexander Berghaus \\ Moritz Gröger \\ Department of Otorhinolaryngology, Head and Neck Surgery, Ludwig Maximilian University Munich, \\ Munich, Germany
}

\section{Key Words}

Allergic rhinoconjunctivitis - Component-resolved diagnostics · Inhalant allergens · Panallergens · Polcalcin · Profilin

\begin{abstract}
Background: Component-resolved diagnostics is gaining importance in allergy diagnostics. Allergen extracts contain components with different rates of prevalence and clinical relevance, which can be subdivided at molecular level into major and minor allergens. Clinical complaints are usually triggered by major allergens, while the role of sensitization to the panallergens profilin and polcalcin still remains unclear. Methods: Eighty-six patients from southern Bavaria with sensitization to the panallergens profilin (Bet $v 2 / \mathrm{Phl} p$ 12) and/or polcalcin (Bet $v$ 4/Phl $p$ 7) were examined in regard to their sensitization to the 4 main botanic denominations Betulaceae, Oleaceae, Poaceae and Asteraceae by skin prick test and measurement of specific immunoglobulin $E$ antibodies to natural allergen extracts as well as major allergen components rPhl p 1/5, rBet v 1, rOle e 1 and nArt v 1 . Sensitization was rated as clinically relevant or irrelevant depending on anamnesis or intranasal allergen challenge. Results: Regarding the 4 botanic denominations, there was no sig-
\end{abstract}

nificant difference in the incidence of sensitization to the panallergens profilin, polcalcin or both. The sensitization pattern does not alter when subdividing the cohort into clinically relevant and silent sensitization. We did not find clinically symptomatic sensitization to panallergens without cosensitization to a major allergen. Conclusions: Our results suggest that sole sensitization to panallergens seems to have no clinical relevance in allergic rhinoconjunctivitis. Clinical complaints seem to be triggered manly by major allergens. Thus, component-resolved allergy diagnostics is crucial in the diagnosis and treatment of polysensitized patients.

(c) 2016 S. Karger AG, Basel

\section{Introduction}

Component-resolved diagnostics (CRD) is considered the state-of-the-art diagnostic approach to identify allergen sensitization of a patient at molecular level $[1,2]$. Pollen extracts contain a variety of allergens, which differ in their allergenicity. By purifying specific immunoglobulin E (IgE)-binding proteins from pollen extracts or by recombinant protein expression in Escherichia coli, single allergens can be identified and analyzed structurally and immunologically. Therefore, CRD based on single re-

\section{KARGER}

E-Mail karger@karger.com

www.karger.com/iaa
(C) 2016 S. Karger AG, Basel

$1018-2438 / 16 / 1692-0101 \$ 39.50 / 0$
Correspondence to: Dr. med. Marion San Nicoló

Department of Otorhinolaryngology, Head and Neck Surgery

Ludwig Maximilian University Munich

Marchioninistrasse 15, DE-81377 Munich (Germany)

E-Mail Marion.Sannicolo@med.uni-muenchen.de 
combinant or natural allergen molecules enables a precise diagnosis of individual sensitization patterns. Symptomeliciting allergens can be distinguished from highly crossreactive allergens in polysensitized patients [3]. CRD, therefore, leads to more accurate allergy diagnosis.

When looking at the sensitization of patients to a certain allergen source, one can distinguish between major and minor allergens. An allergen is considered a major allergen if $50 \%$ or more of the sensitized patients produce specific IgE to that specific allergen. In contrast, a minor allergen is considered as such if fewer patients produce specific IgE [4]. This subdivision is irrespective of its clinical relevance and has to be evaluated regionally.

For inhalant allergens, a wide range of recombinant allergens has been produced over the last years. In this article, we will focus on birch, ash, mugwort and timothy as common inhalant allergens in the western civilization. Major allergens are considered to be the relevant trigger of allergic symptoms in patients [5]. Such major allergens are Bet $\mathrm{v} 1$ (birch), Phl $\mathrm{p} 1$ and 5 (timothy grass), Art v 1 (mugwort plant) and Ole e 1 (olive tree), which is highly homologous to Fra e 1 (ash tree), respectively [6-9]. Some minor allergens are widespread in pollens from different plants and are, therefore, called panallergens. The two most important groups of panallergens are profilin and calcium-binding proteins (polcalcins). Profilin is part of the eukaryotic cytoskeleton [10] and shows great homology throughout different plants. This leads to high crossreactivity of profilins. Diagnostically important profilins are Bet v 2 (birch) and Phl p 12 (timothy grass). Polcalcin participates in the signal transduction pathway in eukaryotic cells by binding to calcium [9]. They are widely distributed proteins in tree, grass and weed pollen [10]. Polcalcins from birch and timothy grass are Bet $\mathrm{v} 4$ and $\mathrm{Phl}$ $\mathrm{p} 7$, respectively. Panallergens are discussed to be a cause of polysensitization in patients [6]. However, the source of sensitization as well as its clinical relevance still remains unclear.

In this study, we investigated the sensitization patterns of pollen-allergic patients sensitized to profilin, polcalcin or both. The study aims to reveal the clinical relevance of these panallergens in our patient cohort.

\section{Patients and Methods}

Patient data were retrospectively selected from the allergy database of the Department of Otorhinolaryngology, Head and Neck Surgery of the Ludwig Maximilian University in Munich storing all relevant diagnostic patient results, including data from skin prick tests (SPTs; ALK-Abelló, Wedel, Germany) to timothy grass, rye, birch, hazel, alder, ash, mugwort, pellitory, ragweed, Dermatophagoides pteronyssinus, Dermatophagoides farinae, dog, cat, Alternaria and Aspergillus. Histamine dihydrochloride solution at $1 \mathrm{mg} / \mathrm{ml}$ was used as positive control and allergen-free saline solution as negative control. SPT was considered positive with a wheal diameter $>3 \mathrm{~mm}$, which was assessed $20 \mathrm{~min}$ after application. The procedure was in line with the literature [11]. The database includes also results from IgE measurements and allergen provocations.

IgE reactivity to purified natural allergen extract and allergen components was measured using the FEIA method (UniCAPFEIA, Thermo Fisher Scientific, Freiburg, Germany) with a commercially available test kid (Phadia Diagnostics, Freiburg, Germany) according to the manufacturer's instructions. Focusing on the 4 main botanic denominations Poaceae, Betulaceae, Oleaceae and Asteraceae, specific IgE antibodies to the native extracts of timothy grass, birch, ash and mugwort were measured in case of a positive SPT result. The panallergens profilin (Bet $\mathrm{v} 2 / \mathrm{Phl} \mathrm{p} \mathrm{12)} \mathrm{and} \mathrm{polcal-}$ cin (Bet v 4/Phl p 7) as well as the major allergen components $\mathrm{rPhl}$ $\mathrm{p} 1 / 5$, rBet $\mathrm{v} 1$, rOle e 1 and nArt $\mathrm{v} 1$ had been determined. Results were reported as CAP classes as well as concentrations (kU/l).

Patients with positive in vivo or in vitro allergy tests do not necessarily suffer from an allergic disease. An accurate medical history is crucial to determine the clinical relevance of the sensitization. Sensitizations were rated as relevant if the patient presented an apparent positive anamnesis for the specific allergen. In case of doubt, a nasal or conjunctival provocation (ALK-Abelló) indicates the clinical relevance of the sensitization to inhalant allergens according to guideline specifications $[12,13]$. The intranasal provocation was rated positive if the rhinomanometry decreased $>40 \%$ at $150 \mathrm{~Pa}$ on the allergen-challenged side or the patient had a symptom score $>3$. It was also rated positive with a decrease in rhinomanometry $>20 \%$ at $150 \mathrm{~Pa}$ on the allergen-challenged side in combination with a symptom score $>2$. Four categories of nasal symptoms were semiquantitatively assessed on the first visit: (1) obstruction, (2) rhinorrhea, (3) sneezing and (4) itching. Each symptom could be classified as: $0=$ no impairment, $1=$ mild impairment, $2=$ moderate impairment and $3=$ severe impairment. Sensitization with unreliable classification because of missing anamnestic information or challenge data was excluded from the study due to its retrospective character, which renders further in vivo data acquisition impossible.

The database was scanned for consecutive patients with a proven sensitization to profilin and/or polcalcin who got extensive allergy diagnostics at our institution between 2009 and 2013. Of 1,145 patients, 86 met the inclusion criteria.

The study was approved by the local ethics committee and the local data protection commissioner. All patients gave informed consent.

\section{Results}

\section{Sensitization Patterns of Major and Minor Allergens}

In our cohort, patients were stratified for all 4 botanic denominations by SPT. For birch $(\mathrm{n}=80)$, ash $(\mathrm{n}=$ $29)$, timothy $(n=85)$ and mugwort $(n=26)$, sensitization to their major allergen Bet v 1, Ole e $1, \mathrm{Phl}$ p $1 / 5$ or 


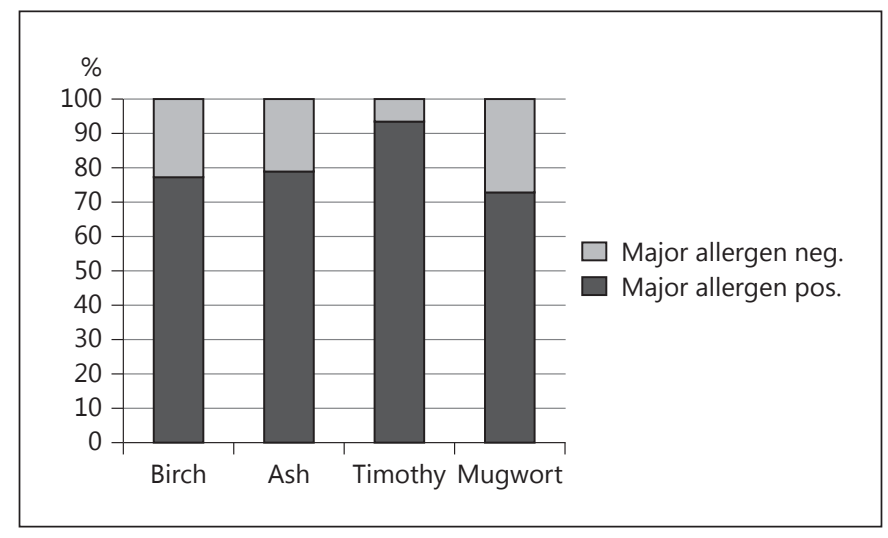

Fig. 1. Sensitization to the major allergen of birch (Bet $v 1$ ), ash (Ole e 1), timothy (Phl p 1/5) and mugwort (Art v 1) in panallergen-sensitized patients.

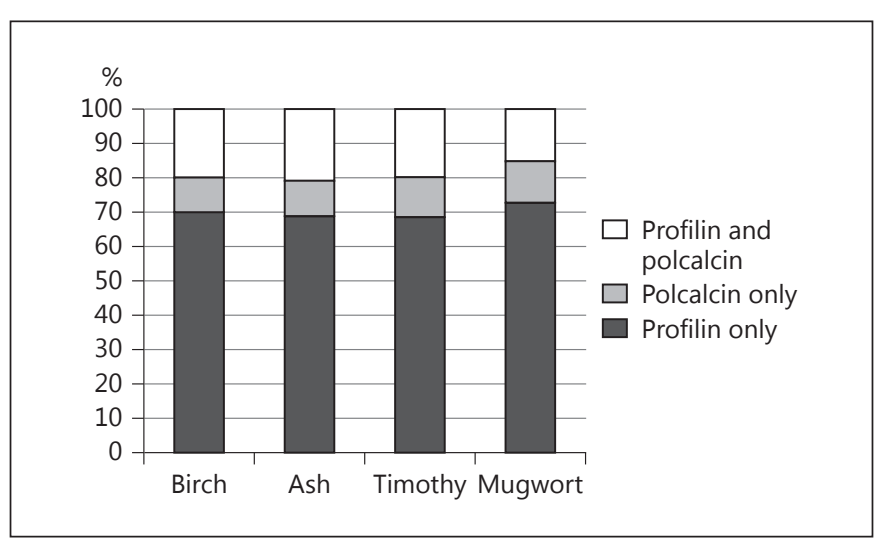

Fig. 2. Stratification by sensitization to the minor allergens profilin and/or polcalcin in panallergen-sensitized patients.

Table 1. Sensitization to the panallergens profilin and polcalcin in major allergen-positive and -negative sensitization

\begin{tabular}{|c|c|c|c|c|c|c|}
\hline $\begin{array}{l}\text { Sensitization } \\
\text { Profilin/polcalcin }\end{array}$ & \multicolumn{3}{|c|}{ Major allergen positive } & \multicolumn{3}{|c|}{ Major allergen negative } \\
\hline Ash & $16(70 \%)$ & $3(13 \%)$ & $4(17 \%)$ & $4(67 \%)$ & 0 & $2(33 \%)$ \\
\hline Timothy & $54(68 \%)$ & $8(10 \%)$ & $17(22 \%)$ & $5(83 \%)$ & $1(17 \%)$ & 0 \\
\hline Mugwort & $14(74 \%)$ & $1(5 \%)$ & $4(21 \%)$ & $5(71 \%)$ & $2(29 \%)$ & 0 \\
\hline
\end{tabular}

nArt v 1, respectively, was examined (fig. 1). Major allergen sensitization clearly predominates in patients sensitized to minor allergens profilin, polcalcin or both: $78 \%$ for Bet v 1, 79\% for Ole e 1, 93\% for Phl p 1/5 and $73 \%$ for nArt v 1 .

When differentiating the sensitization to birch, ash, timothy and mugwort into sole sensitization to profilin or polcalcin or a double sensitization to both panallergens, the following can be shown: Sole profilin sensitization has the highest rate with about $70 \%$ in all groups, followed by the concomitant sensitization of profilin and polcalcin (about 20\%) and the sole sensitization of polcalcin (about 10\%; fig. 2).

Furthermore, we investigated the sensitization of the panallergens profilin and polcalcin within the group of major allergen-positive and -negative sensitization (table 1).

There was a similar sensitization profile to minor allergens in all 4 botanic groups. Profilin clearly predominates the major allergen-positive and -negative group.
Overall, 68\% of major allergen-positive sensitization were found to be cosensitized to profilin. In the major allergennegative group of sensitization, $81 \%$ were sensitized to profilin. Polcalcin sensitization alone was found in $10 \%$ of the patients with major allergen-positive sensitization and in $11 \%$ with major allergen-negative sensitization. A double sensitization to profilin and polcalcin was found in a total of $22 \%$ of major allergen-positive and $8 \%$ of major allergen-negative sensitization results.

\section{Clinical Relevance of Panallergens}

Figure 3 shows the distribution of clinically irrelevant versus relevant sensitization in our cohort. Timothy grass had the highest amount of clinically relevant sensitization with $64 \%$, followed by birch (46\%), ash (38\%) and mugwort (19\%).

We further stratified the group with (fig. 4a) and without (fig. 4b) clinically relevant sensitization to birch, ash, timothy and mugwort by their expression of IgE against the panallergens profilin and polcalcin. 


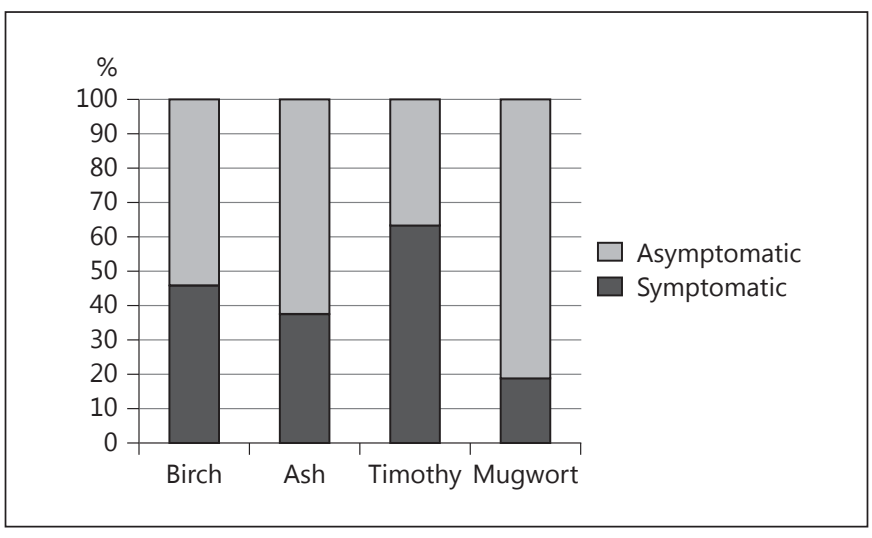

Fig. 3. Clinical relevance of sensitization to inhalant allergens (in patients sensitized to profilin and/or polcalcin). The black bars illustrate the percentage of sensitizations with clinical relevance, and the gray bars sensitizations without clinical relevance.

The expression patterns in both cohorts indicate similar results regarding the single components. Profilin clearly predominates in clinically relevant and silent sensitization in all 4 botanic denominations with an IgE expression of $62-81 \%$, followed by the concomitant IgE expression of both panallergens in $0-24 \%$ and the sole IgE expression for polcalin in 6-20\%.

To further investigate the clinical relevance of sensitization to panallergens, we conducted an analysis of symptomatic and asymptomatic sensitization in regard to their cosensitization to major allergens (table 2). In our study, no major allergen-negative sensitization showed to be symptomatic. The sensitization patterns to panallergens in asymptomatic sensitization showed to be similar in major allergen-positive and -negative sensitization (table 2).

\section{Discussion}

CRD presents a precise method for individual allergy diagnostics. It is now possible to differentiate a patient's specific sensitization pattern instead of dealing with natural pollen extracts containing allergens of different allergenicity as well as other proteins and carbohydrates in poorly defined concentrations [14] leading to a lack of analytical specificity. Thus, a more accurate and individual identification of allergen profiles can be achieved and disease-eliciting allergen components might be differentiated from confounders.

By implementing molecular allergy diagnostics, a distinction between major and minor allergens was possible.

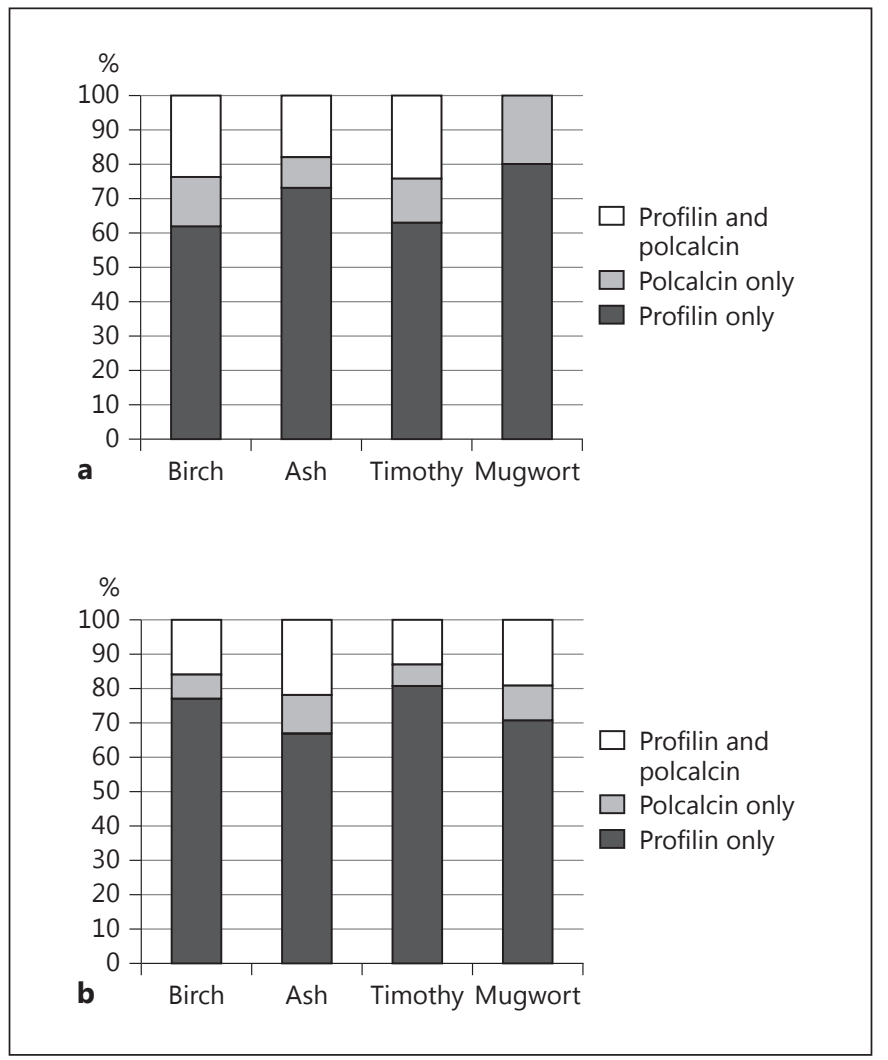

Fig. 4. Sensitization to panallergens (profilin/polcalcin) in clinically relevant (a) and silent (b) sensitizations to birch, ash, timothy grass or mugwort.

Major allergens are considered as triggers of clinically relevant allergy in most cases. Minor allergens are considered as markers of multiple pollen sensitization [15]. Profilins and calcium-binding proteins (polcalcins) are widespread even in botanically unrelated sources and, therefore, presumed to provoke a high potential of allergenic cross-reactivity $[16,17]$. This cross-reactivity is discussed to be a reason for nonresponse in allergen-specific immunotherapy (AIT). The average success rate of AIT ranges from 80 to $85 \%$ in pollen-sensitive patients [18]. As clinical symptoms in patients seem to be triggered by major allergen components, the success of immunotherapies might be largely determined by major allergens [6]. Commercially available immunotherapy solutions are standardized for their content of major allergens only. Therefore, a therapeutic dosage can only be guaranteed for major allergens. Furthermore, especially in patients sensitized to multiple, taxonomically unrelated allergens, the clinician has to determine whether sensitization is due to cosensitization to different 'genuine' allergens or due 
Table 2. Sensitization patterns to panallergens and major allergens in clinically symptomatic and asymptomatic sensitization to birch, ash, timothy grass or mugwort

\begin{tabular}{|c|c|c|c|c|c|c|}
\hline Profilin/polcalcin & \multicolumn{3}{|c|}{ Major allergen positive } & \multicolumn{3}{|c|}{ Major allergen negative } \\
\hline \multicolumn{7}{|c|}{ Symptomatic sensitization } \\
\hline Birch & $23(62 \%)$ & $5(14 \%)$ & $9(24 \%)$ & 0 & 0 & 0 \\
\hline Ash & $8(73 \%)$ & $1(9 \%)$ & $2(18 \%)$ & 0 & 0 & 0 \\
\hline Timothy & $34(63 \%)$ & $7(13 \%)$ & $13(24 \%)$ & 0 & 0 & 0 \\
\hline Total & $69(65 \%)$ & $14(13 \%)$ & $24(22 \%)$ & 0 & 0 & 0 \\
\hline \multicolumn{7}{|c|}{ Asymptomatic sensitization } \\
\hline Birch & $17(68 \%)$ & $2(8 \%)$ & $6(24 \%)$ & $16(89 \%)$ & $1(6 \%)$ & $1(6 \%)$ \\
\hline Ash & $8(67 \%)$ & $2(17 \%)$ & $2(17 \%)$ & $4(67 \%)$ & 0 & $2(33 \%)$ \\
\hline Timothy & $20(80 \%)$ & $1(4 \%)$ & $4(16 \%)$ & $5(83 \%)$ & $1(17 \%)$ & 0 \\
\hline
\end{tabular}

to a corecognition of homologous panallergens [19]. Thus, a sensitization to panallergens might explain at least part of the $15-20 \%$ who are not responding. Sole sensitization to panallergens can often not be attributed to a specific allergen source and is, therefore, not suited for AIT [20]. But how clinically relevant is a sensitization to panallergens?

To date, sensitization to panallergens is mainly detected by IgE reactivity in vitro. Even though SPT solutions with profilin and polcalcin have been developed [21,22] and used as provocation agents [23, 24], larger studies proving the clinical relevance of panallergens are lacking to date.

Sensitization and immunological cross-reactivity do not predict a clinically relevant allergy. Scheurer et al. [25] found strong IgE reactivity to recombinant plant food profilins in Bet v 2-sensitized patients. Interestingly, these patients presented no clinical symptoms. Similar results were found by Wensing et al. [26] and Ebo et al. [27], suggesting a minor relevance of sole profilin sensitization in triggering relevant food allergies. As a food allergen, profilin is actually described to trigger merely an oral allergy syndrome but it seems also able to induce severe reactions $[28,29]$. Several authors assume that a high rate of digestion by pepsin in the gastric fluid leads to a reduced enzymatic stability. This, as well as low thermal stability, results in only mild allergic symptoms like the oral allergy syndrome [25, 30]. Fah et al. [31] reported a case of anaphylactic reaction to lychee fruit. IgE to profilin in the patient's serum was the only clinical finding. The authors discussed that the high level of profilin might have overcome the capacity of gastric protein digestion and therefore led to a high absorption of profilin, which again triggered anaphylaxis.

However, the clinical relevance of sensitization to panallergens remains subject to controversy until a reliable in vivo provocation test is available. There is only slight evidence that profilin elicits nasal and bronchial responses. Especially nasal responses to profilin are quite weak [23, 24]. In this retrospective study, we analyzed the sensitization patterns of pollen-sensitized patients in southern Bavaria who were positive to panallergens. The incidence of sensitization to profilin and polcalcin as well as to major allergens was examined in panallergen-positive patients and their clinical relevance was assessed. Profilin was found to be the most common minor allergen in our patients, who showed IgE expression against profilin only in about $70 \%$ of the cases. These data are in agreement with previous studies [6].

We found that sole sensitization to minor allergens seems to have no clinical relevance in allergic rhinoconjunctivitis. Clinical complaints seem to be triggered by the major allergens. $100 \%$ of the patients with clinical complaints showed to be IgE positive for the specific major allergen component. In cases of sensitization without clinical symptoms, a consistent distribution of sensitization patterns to panallergens was seen irrespective of cosensitization to major allergens such as birch, ash, timo- 
thy grass or mugwort. In case of clinically relevant sensitization, the sensitization pattern to panallergens was found be similar to that to major allergens only. Symptomatic but major allergen-negative sensitization could not be detected. In our study, clinical symptoms were always accompanied by sensitization to major allergens whereas a major allergen sensitization on its own did not imply clinical relevance.

In a cross-sectional study, Scala et al. [32] analyzed the molecular profiles of over 23,000 patients in relation to age. A clear difference in the stratified groups was found. Melioli et al. [33] confirmed this by showing a higher rate of overall sensitization in adolescence than in childhood. Young children presented sensitization to food allergens while 'genuine' components of grass, Parietaria and birch were rare before the age of 3 years. Interestingly, crossreacting allergens were almost absent in the first years of childhood.

The term 'molecular spreading' is used to describe the recent finding of an evolution of sensitization - from molecular monosensitization in early childhood to oligoand polysensitization in adolescence and adulthood [34]. This could indicate that sensitization to panallergens follows a sensitization to the corresponding major allergen over time. If sensitization to panallergens follows the sensitization to the specific major allergen in a chronological order, major allergen sensitization should predominantly be seen in polysensitized patients and, therefore, allow to draw conclusions on the origin of the panallergen sensitization [35]. Looking at our data, rates of sensitization to specific major allergens in panallergen-sensitized patients correspond approximately in all 4 botanic groups. Remarkably, 85 of the 86 patients were sensitized to grass pollen, 79 (93\%) of these grass pollen-sensitized subjects were $\mathrm{Phl} \mathrm{p} \mathrm{1/5} \mathrm{positive.} \mathrm{This} \mathrm{is} \mathrm{in} \mathrm{line} \mathrm{with} \mathrm{observations}$ from others describing an interrelationship of profilin sensitization with grass allergy $[29,36]$. Within the other 3 botanic groups, sensitization rates to the particular major allergen ranged between 73 and 79\%. Hence, we cannot definitely deduce the primary source of sensitization to panallergens; this could be an indication of the route via grass pollen [22].

Thus, component-resolved allergy diagnostics is important in the diagnosis of polysensitized patients. In order to avoid nonresponse to AIT and a possible iatrogenic sensitization [2], the proof of major allergen components is crucial.

Further investigations, especially the introduction of an in vivo provocation test with profilin and polcalcin as the gold standard in allergy diagnostics, need to be advanced to gain more insight into the role of minor allergens in allergic rhinoconjunctivitis.

\section{References}

1 Valenta R, Lidholm J, Niederberger V, Hayek B, Kraft D, Gronlund H: The recombinant allergen-based concept of component-resolved diagnostics and immunotherapy (CRD and CRIT). Clin Exp Allergy 1999;29: 896-904.

2 Canonica GW, Ansotegui IJ, Pawankar R, et al: A WAO - ARIA - GA²LEN consensus document on molecular-based allergy diagnostics. World Allergy Organ J 2013;6:17.

3 Canis M, Groeger M, Becker S, Klemens C, Kramer MF: Recombinant marker allergens in diagnosis of patients with allergic rhinoconjunctivitis to tree and grass pollens. Am J Rhinol Allergy 2011;25:36-39.

4 Marsh DG, Goodfriend L, King TP, Lowenstein H, Platts-Mills TA: Allergen nomenclature. Bull World Health Organ 1986;64:767774.

5 Schmid-Grendelmeier P: Recombinant allergens. For routine use or still only science (in German)? Hautarzt 2010;61:946-953.

6 Muehlmeier G, Maier H: Polysensitisation to pollen due to profilin and calcium-binding protein: distribution of $\mathrm{IgE}$ antibodies to marker allergens in grass and birch pollen al- lergic rhinitis patients in southern Germany. Eur Arch Otorhinolaryngol 2014;271:719725.

7 Palomares O, Swoboda I, Villalba M, et al: The major allergen of olive pollen Ole e 1 is a diagnostic marker for sensitization to Oleaceae. Int Arch Allergy Immunol 2006;141:110-118.

8 Leonard R, Wopfner N, Pabst M, et al: A new allergen from ragweed (Ambrosia artemisiifolia) with homology to Art v 1 from mugwort. J Biol Chem 2010;285:27192-27200.

9 Wopfner N, Gadermaier G, Egger M, et al: The spectrum of allergens in ragweed and mugwort pollen. Int Arch Allergy Immunol 2005;138:337-346.

10 Valenta R, Duchene M, Ebner C, et al: Profilins constitute a novel family of functional plant pan-allergens. J Exp Med 1992;175:377385.

11 Heinzerling L, Frew AJ, Bindslev-Jensen C, et al: Standard skin prick testing and sensitization to inhalant allergens across Europe - a survey from the GALEN network. Allergy 2005;60:1287-1300.

12 Cazan D, Hackenberg B, Pfaar O, Klimek L: Die nasale Provokationstestung mit Aller- genen - Methoden der klinischen Anwendung. Allergo J 2013;22:189-200.

13 Riechelmann H, Bachert C, Goldschmidt O, et al: Application of the nasal provocation test on diseases of the upper airways. Position paper of the German Society for Allergology and Clinical Immunology (ENT Section) in cooperation with the Working Team for Clinical Immunology (in German). Laryngorhinootologie 2003;82:183-188.

14 The use of standardized allergen extracts. American Academy of Allergy, Asthma and Immunology (AAAAI). J Allergy Clin Immunol 1997;99:583-586.

15 Mari A: Multiple pollen sensitization: a molecular approach to the diagnosis. Int Arch Allergy Immunol 2001;125:57-65.

16 Vieths S, Scheurer S, Ballmer-Weber B: Current understanding of cross-reactivity of food allergens and pollen. Ann NY Acad Sci 2002; 964:47-68.

17 Bonura A, Gulino L, Trapani A, et al: Isolation, expression and immunological characterization of a calcium-binding protein from Parietaria pollen. Mol Immunol 2008;45: 2465-2473. 
18 Calderon MA, Alves B, Jacobson M, Hurwitz B, Sheikh A, Durham S: Allergen injection immunotherapy for seasonal allergic rhinitis. Cochrane Database Syst Rev 2007;1: CD001936.

19 Ferreira F, Hawranek T, Gruber P, Wopfner $\mathrm{N}$, Mari A: Allergic cross-reactivity: from gene to the clinic. Allergy 2004;59:243-267.

20 Gangl K, Niederberger V, Valenta R, Nandy A: Marker allergens and panallergens in tree and grass pollen allergy. Part 17 of the Series Molecular Allergology (in German). Allergo J Int 2015;24:158-169.

21 Barber D, de la Torre F, Lombardero M, et al: Component-resolved diagnosis of pollen allergy based on skin testing with profilin, polcalcin and lipid transfer protein pan-allergens. Clin Exp Allergy 2009;39:1764-1773.

22 Asero R, Jimeno L, Barber D: Preliminary results of a skin prick test-based study of the prevalence and clinical impact of hypersensitivity to pollen panallergens (polcalcin and profilin). J Investig Allergol Clin Immunol 2010;20:35-38.

23 Ruiz-Garcia M, Garcia Del Potro M, Fernandez-Nieto M, Barber D, Jimeno-Nogales L, Sastre J: Profilin: a relevant aeroallergen? J Allergy Clin Immunol 2011;128:416-418.
24 Asero R, Villalta D: Profilin may be a primary airborne sensitizer: a case report. J Investig Allergol Clin Immunol 2013;23:134-135.

25 Scheurer S, Wangorsch A, Nerkamp J, et al: Cross-reactivity within the profilin panallergen family investigated by comparison of recombinant profilins from pear (Pyr c 4), cherry (Pru av 4) and celery (Api g 4) with birch pollen profilin Bet v 2. J Chromatogr B Biomed Sci Appl 2001;756:315-325.

26 Wensing $\mathrm{M}$, Akkerdaas $\mathrm{JH}$, van Leeuwen WA, et al: IgE to Bet $\mathrm{v} 1$ and profilin: crossreactivity patterns and clinical relevance. J Allergy Clin Immunol 2002;110:435-442.

27 Ebo DG, Hagendorens MM, Bridts CH, De Clerck LS, Stevens WJ: Sensitization to crossreactive carbohydrate determinants and the ubiquitous protein profilin: mimickers of allergy. Clin Exp Allergy 2004;34:137-144.

28 Asero R, Monsalve R, Barber D: Profilin sensitization detected in the office by skin prick test: a study of prevalence and clinical relevance of profilin as a plant food allergen. Clin Exp Allergy 2008;38:1033-1037.

29 Alvarado MI, Jimeno L, De La Torre F, et al: Profilin as a severe food allergen in allergic patients overexposed to grass pollen. Allergy 2014;69:1610-1616.

30 Rodriguez-Perez R, Crespo JF, Rodriguez J, Salcedo G: Profilin is a relevant melon allergen susceptible to pepsin digestion in patients with oral allergy syndrome. J Allergy Clin Immunol 2003;111:634-639.
31 Fah J, Wuthrich B, Vieths S: Anaphylactic reaction to lychee fruit: evidence for sensitization to profilin. Clin Exp Allergy 1995;25: 1018-1023.

32 Scala E, Alessandri C, Bernardi ML, et al: Cross-sectional survey on immunoglobulin $\mathrm{E}$ reactivity in 23,077 subjects using an allergenic molecule-based microarray detection system. Clin Exp Allergy 2010;40:911-921.

33 Melioli G, Marcomini L, Agazzi A, et al: The IgE repertoire in children and adolescents resolved at component level: a cross-sectional study. Pediatr Allergy Immunol 2012;23:433440

34 Matricardi PM: Molecular profile clustering of IgE responses and potential implications for specific immunotherapy. Curr Opin Allergy Clin Immunol 2013;13:438-445.

35 Barber D, de la Torre F, Feo F, et al: Understanding patient sensitization profiles in complex pollen areas: a molecular epidemiological study. Allergy 2008;63:1550-1558.

36 Asero R, Jimeno L, Barber D: Preliminary results of a skin prick test-based study of the prevalence and clinical impact of hypersensitivity to pollen panallergens (polcalcin and profilin). J Investig Allergol Clin Immunol 2010;20:35-38. 